\title{
Erratum to: Pressure-Loaded MSCs During Early Osteodifferentiation Promote Osteoclastogenesis by Increase of RANKL/OPG Ratio
}

\author{
Jun Liu, ${ }^{1}$ Zhihe Zhao, ${ }^{1}$ Ling Zou, ${ }^{1}$ Juan Li ${ }^{1}$ Fengming Wang, ${ }^{1}$ Xiaoyu Li, ${ }^{1}$ Jingyi Zhang, ${ }^{1}$ \\ Yurong Liu, ${ }^{1}$ Sixiu Chen, ${ }^{1}$ Maohui Zhi, ${ }^{2}$ and Jun Wang ${ }^{1}$
}

${ }^{1}$ State Key Laboratory of Oral Diseases, West China College of Stomatology, West China Hospital of Stomatology, Sichuan University, 14\#, 3rd section, Renmin South Road, Chengdu 610041, China; and ${ }^{2}$ Laboratory of Functional Science, West China School of Basic Medicine and Forensic Sciences, Sichuan University, Chengdu, China

Erratum to: Annals of Biomedical Engineering (2009) 37(4):794-802

DOI 10.1007/s10439-009-9638-9

The authors wish to replace Figure 1 in Ann Biomed Eng, 2009; 37:794-802 with the current figure and legend, as shown below. In the original paper, the authors failed to properly attribute the source of the data shown in the figure and corresponding text used in the Introduction section. The data and figure, which were used as background information, had been taken from the following article published by the same authors:

Hydrostatic pressure promotes Wnt10b and Wnt4 expression dependent and independent on ERK signaling in early-osteoinduced MSCs (2009) Jun Liu, Ling Zou, Jun Wang, Charles Schuler, Zhihe Zhao, Xiaoyu Li, Jingyi Zhang, and Yurong Liu, Biochemical and Biophysical Research Communications, 379:505-509.

This is the figure and legend as they should have appeared in the Annals of Biomedical Engineering.

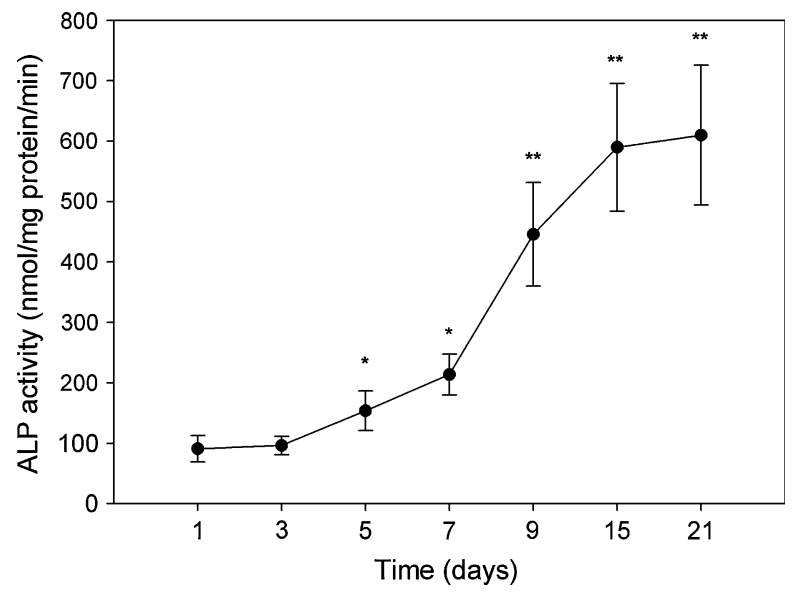

FIGURE 1. Alkaline phosphatase (ALPase) activity in osteoinduced rat MSCs over 21 days $\left({ }^{*} p<0.05\right.$ and $\left.{ }^{* *} p<0.01\right)$. We aimed to examine MSCs during their initial osteodifferentiation. Therefore, we used MSCs at the stages of 0-, 3- and 7-day-culture (OS-0d, OS-3d and OS-7d) which expressed low levels of ALPase activity. Data from Liu et al., Biochemical and Biophysical Research Communications, 379:505-509, 2009.

Address correspondence to Jun Wang, State Key Laboratory of Oral Diseases, West China College of Stomatology, West China Hospital of Stomatology, Sichuan University, 14\#, 3rd section, Renmin South Road, Chengdu 610041, China. Electronic mail: wangjunv@yahoo.cn

The online version of the original article can be found under doi: 10.1007/s10439-009-9638-9. 